\title{
Influência dos atributos físicos do solo na produção e qualidade do algodão em
}

\section{caroço e fibra}

\author{
Influence of soil physical attributes on the production and quality of the cotton in seed and fiber \\ Influencia de los atributos físicos del suelo en la producción y calidad del algodón en semillas y
}

fibras

Recebido: 18/10/2021 | Revisado: 28/10/2021 | Aceito: 01/11/2021 | Publicado: 03/11/2021

\author{
Pâmila Nayana Ferreira Ramos \\ ORCID: https://orcid.org/0000-0003-3027-4515 \\ Universidade Federal de Mato Grosso, Brasil \\ E-mail: pamilanayana@yahoo.com.br \\ Paulo Afonso Ferreira \\ ORCID: https://orcid.org/0000-0001-8054-4072 \\ Universidade Federal de Mato Grosso, Brasil \\ E-mail: pafonsoferreira@yahoo.com.br \\ Omar Roberto da Silveira \\ ORCID: https://orcid.org/0000-0002-1556-4808 \\ Universidade Federal de Mato Grosso, Brasil \\ E-mail: omar.silveira@agricultura.gov.br \\ João Carlos de Souza Maia \\ ORCID: https://orcid.org/0000-0001-6204-8112 \\ Universidade Federal de Mato Grosso, Brasil \\ E-mail: jotace@terra.com.br
}

\begin{abstract}
Resumo
A produção de algodão representa uma importante cultura agrícola do Brasil. Portanto se faz necessário estudos que relacionam atributos físicos do solo com produção de fibra e produção de algodão em caroço. Sendo assim, este trabalho teve como objetivo determinar a influência dos atributos físicos do solo: argila, areia, silte, microporosidade, macroporosidade, porosidade total e densidade, na forma colhida do algodão. Realizamos análise de correlação canônica, utilizando os dados amostrais obtidos de 193 amostras de solo, coletadas em 100 talhões com 13 cultivares de algodão, entre 63 e 136 dias após a emergência, distribuídos em 3 regiões do estado de Mato Grosso, em altitudes de 402 a $885 \mathrm{~m}$. A análise de correlação canônica explicou 82,99\% da variação dos dados amostrais. Os atributos físicos do solo influenciaram na produção do algodoeiro, no rendimento de fibra e na produção de algodão em caroço, nesta ordem: $($ Silte $=$ Microporosidade $)>($ Argila $=$ Macroporosidade $=$ Areia $)>>$ microporosidade, porosidade Total $>$ Densidade. Os atributos densidade, porosidade total e macroporosidade não afetaram na produção de fibra, respectivamente. Neste sentido os atributos físicos do solo alteram a qualidade da fibra.
\end{abstract}

Palavras-chave: Quantitativos; Resistência; Variáveis; Agricultura.

\begin{abstract}
Cotton production represents an important agricultural crop in Brazil. Therefore, studies associating soil physical attributes with fiber and seed cotton production are necessary. Accordingly, this work aimed to determine the influence of soil physical attributes: clay, sand, silt, microporosity, macroporosity, total porosity and density, in the harvested form of cotton. A canonical correlation analysis was carried out using data obtained from 193 soil samples that were collected in 100 plots with 13 cotton cultivars, between 63 and 136 days after emergence, distributed in 3 regions of the State of Mato Grosso, at altitudes of between 402 and $885 \mathrm{~m}$. The canonical correlation analysis explained $82.99 \%$ of the sample data variation. The soil physical attributes influenced in the cotton plant production, fiber yield and seed cotton production, in this sequence: $($ Silt $=$ Microporosity $)>($ Clay $=$ Macroporosity $=$ Sand $)>>$ microporosity, Total porosity > Density. The density, total porosity and macroporosity did not affect fiber production, respectively. In this regard, soil physical attributes alter the fiber quality.
\end{abstract}

Keywords: Quantitative; Resistance; Variables; Agriculture.

\section{Resumen}

La producción de algodón representa un cultivo agrícola importante en Brasil. Por lo tanto, se necesitan estudios que relacionen los atributos físicos del suelo con la producción de fibra y la producción de algodón en rama. Así, este trabajo tuvo como objetivo determinar la influencia de los atributos físicos del suelo: arcilla, arena, limo, 
microporosidad, macroporosidad, porosidad total y densidad, en la forma cosechada del algodón. Realizamos análisis de correlación canónica, utilizando datos de muestra obtenidos de 193 muestras de suelo, recolectadas en 100 parcelas con 13 cultivares de algodón, entre 63 y 136 días después de la emergencia, distribuidas en 3 regiones del estado de Mato Grosso, en altitudes de 402 a 885m. El análisis de correlación canónica explicó el 82,99\% de la variación de los datos de la muestra. Los atributos físicos del suelo influyeron en la producción de algodón y el rendimiento de fibra y la producción de semilla de algodón, en este orden: $($ Limo $=$ Microporosidad $)>($ Arcilla $=$ Macroporosidad $=$ Arena $)$ > Microporosidad, Porosidad total> Densidad. Los atributos densidad, porosidad total y macroporosidad no afectaron la producción de fibra, respectivamente. En este sentido, los atributos físicos del suelo alteran la calidad de la fibra.

Palabras clave: Cuantitativo; Resistencia; Variables; Agricultura.

\section{Introdução}

No Brasil a produção de algodão iniciou-se na década de 1990 nas regiões Sul, Sudeste e Nordeste. O aumento na busca da fibra de algodão alterou o cenário e o Brasil se consolida internacionalmente como um grande produtor e exportador dentre os mais de 80 países envolvidos na cadeia produtiva (USDA-FAS, 2018). A produção brasileira de pluma de algodão, na safra 2019/2020, alcançou cerca de 2,9 milhões de toneladas, das quais 67\% foram destinadas à exportação (Shahbandeh, 2019).

Atualmente o nosso país alcançou lugar de destaque nas últimas décadas, principalmente o estado de Mato Grosso, onde o principal responsável pelo sucesso é o algodoeiro que pertence ao gênero Gossypium, da família Malvaceae. A espécie Gossypium hirsutum L. é responsável por $90 \%$ da produção mundial de fibras (Galbieri et al., 2009). O cultivo do algodão destaca-se na agricultura mundial pela utilização dos seus produtos e subprodutos, a cotonicultura é de grande importância para a economia brasileira. O algodoeiro é uma das mais tradicionais e importantes culturas agrícolas para a humanidade, que produziu 1,9 milhões de toneladas de fibra na safra 2019/2020 (Statista, 2020).

A cultura de algodão foi difundida ao longo dos anos no Brasil e atualmente o Mato Grosso, se destacou na produção de algodão, com área de cultivo superior a 1 milhão de hectares e produção de 1,8 milhões de toneladas de pluma, seguido pela Bahia, que cultivou 332 mil hectares e produziu 597 mil toneladas de algodão em pluma Segundo a CONAB (2020) na safra 2018/2019. Em Mato Grosso, a produção de algodão está distribuída nas seguintes regiões: Centro, Centro-Leste, MédioNorte, Noroeste, Norte e Sul. Esta pesquisa foi realizada nas regiões Médio-Norte, Centro e Sul, devido às marcantes diferenças climáticas e de altitude

Aumentando significativamente a participação do algodão produzido nas áreas de cerrado, principalmente na região Centro-Oeste. Com condições favoráveis para o desenvolvimento da cultura e, principalmente e investimentos em pesquisa, no melhoramento genético, que apresente elevada produtividade, o que permitiu obter variedades adaptadas (Smith, 2015).

Com relevo adequado à colheita mecanizada, resistentes a pragas e doenças, com alta adaptação às condições edafoclimáticas do cerrado, alta produtividade tanto em sementes quanto em fibra, aliadas às modernas técnicas de cultivos. O modelo estabelecido no estado, o algodoeiro é utilizado, na sua maioria, em sucessão após a cultura da soja em um mesmo ano agrícola (IMEA, 2016). A produção em grande escala é resultado de pesquisas que avaliaram além do melhoramento genético de plantas e sementes como também estudo de relações entre os atributos físicos do solo.

Várias pesquisas concluíram que atributos físico do solo influenciam na produção de fibra, rendimento de fibra e produção de algodão em caroço, (Galbieri \& Belot, 2016; Barbosa \& Asmus, 2019; Neto, 2019), textura (Holguin et al., 2015; Kumar et al., 2017; Kim et al., 2017), porosidade total, macroporosidade e microporosidade (Stolf et al., 2011; Cardoso et al., 2015; Ortiz et al., 2017), por sua vez a ausência dos atributos físicos do solo, alteram a absorção de nutrientes pelo algodoeiro (Hurchanik et al., 2003; Verma \& Jain, 2006; Jagadeesh \& Gaur, 2014; Jagadeesh \& Gaur, 2014). Também, os nutrientes foliares afetam a fibra, na produção (Tsialtas et al., 2016; Haliloglu, 2019); no rendimento (Eleyan et al., 2014) e na qualidade 
(Afzal et al., 2019; Karademir \& Karademir, 2019; Echer et al., 2020). Entretanto, é preciso estudo de relações entre os atributos físicos do solo para determinar a influência dessa relação na qualidade da produção da fibra, rendimento de fibra e produção de algodão em caroço, através dos teores nutricionais do algodoeiro. Além disso, o estudo foi realizado nas regiões e condições de cultivo do algodoeiro. Com essas premissas, este trabalho teve como objetivo determinar, em áreas de produção de algodão de Mato Grosso: (i) a influência de 7 atributos físicos na qualidade da fibra do algodão, nos teores de nutrientes foliares do algodoeiro; (ii) a influência de 13 nutrientes foliares, na produção e no rendimento de fibra de algodão; e (iii) a influência de 13 nutrientes foliares, em 10 atributos de qualidade da fibra de algodão.

\section{Metodologia}

\section{1 Área de estudo}

Amostramos 100 talhões de cultivo de algodoeiro, em Mato Grosso, nos municípios de Pedra Preta, Nova Mutum e Campo Verde/ MT- Brasil, em 11 fazendas, com altitudes variando de 402 a 885m, no ano de 2019. Cada talhão foi amostrado em local de plantas algodoeiras. Os talhões continham lavoura de algodão com 63 a 136 dias após a emergência, das cultivares FM 954 GLT, FM 906 GLT, FM 913 GLT, FM 944 GL, FM 975 WS, FM 983 GLT, FM 985 GLTP, IMA 2106 GL, IMA 5801 B2RF, TMG 44 B2RF, TMG 45 B2RF, TMG 47 B2RF e TMG 81 WS, (Galbieri et al., 2020).

\subsection{Coleta e análise dos dados}

Cada local de amostragem foi definido como 10 linhas de lavoura de algodão de $10 \mathrm{~m}$ de comprimento, onde colhemos, conforme (Goulart, 2009): (i) uma amostra composta de 5 subamostras de solo e raízes, colhidas na rizosfera de plantas algodoeiras, na profundidade de 0,0 a 0,2 m, para determinação dos teores de areia, silte e argila (amostra de solo): (ii) duas amostras indeformadas de solo para determinação de macroporosidade, microporosidade, porosidade total e densidade, na profundidade de 0,1 a $0,2 \mathrm{~m}$.

As análises de atributos físicos do solo se basearam nos métodos da Embrapa (Teixeira et al., 2017). No entanto, as análises de densidade, macroporosidade, microporosidade e porosidade total sofreram adaptação metodológica. O volume de cada anel de Kopech foi completado com areia fina, utilizando proveta de $10 \mathrm{~mL}$, para medição da areia consumida, com o objetivo de descontar o volume de areia consumida do volume do anel. Essa adaptação conferiu precisão às análises laboratoriais, que dependem do real volume de solo de cada anel.

Para análise do algodoeiro foram, coletadas duas amostras em campo nas três as distintas citadas a cima: (i) uma amostra composta pelas quintas folhas de 5 plantas algodoeiras, contadas a partir do ápice das planta (Kurihara et al., 2014), para análise de $\mathrm{N}$ (nitrogênio), $\mathrm{P}$ (fósforo), $\mathrm{K}$ (potássio), Ca (cálcio), Mg (magnésio), $\mathrm{S}$ (enxofre), Cu (cobre), B (boro), Fe (ferro), Zn (zinco), Mn (manganês), Mo (molibdênio) e Ni (níquel); (ii) uma amostra composta pelo algodão em caroço, produzido pelas 4 plantas algodoeiras mais próximas de cada uma das 5 plantas algodoeiras amostradas para análise foliar, colhido manualmente, durante o período da pré-colheita, para determinação da produção de algodão em caroço, bem como do rendimento, da produção e da qualidade, da fibra. Foram contados os capulhos e as maçãs das 20 plantas algodoeiras. A massa por capulho de algodão foi extrapolada como massa por maçã, já que as maçãs produziriam capulhos até à colheita. Em seguida, para obtenção da massa de algodão em caroço de cada amostra composta, foram somados os números de capulho e de maçã, cuja soma foi multiplicada pela massa de capulho de algodão.

As análises de nutrientes foliares do algodoeiro foram realizadas segundo a metodologia proposta por Malavolta et al. (1997). Os seguintes atributos de qualidade da fibra foram determinados, através de High Volume Instrument (HVI): cor (Cor), maturidade (MAT), finura (MIC), índice de fiabilidade (SCI), índice de fibras curtas (SFI), alongamento até à sua ruptura 
(ELG), reflectância (RD), uniformidade de comprimento (UNF), grau de amarelamento (+b), resistência à ruptura (STR) e comprimento médio (UHM).

Realizamos análise de correlação canônica, que serve para identificar e quantificar a associação entre dois grupos de variáveis (Hotelling, 1935, 1936), utilizando o programa PAST 4.0 (PAleontological STatistics). Os critérios de interpretação gráfica de correlação canônica possibilitaram diferenciação das variações de influência de variável preditora, nas variáveis preditas. Então, estratificamos em três níveis (alto, médio e baixo) a variação da influência de cada variável preditora entre as variáveis preditas.

\section{Resultados}

\subsection{Estatística Descritiva}

A análise de correlação demonstrou que os atributos físicos do solo explicaram as variações na produção do algodão, enquanto que os atributos físicos do solo se destacaram pelas menores variações. A seguir, apresentamos a sequência de variáveis pelo grau de aproximação dos seus dados à curva de normalidade (Macroporosidade (Mac) = Microporosidade (Mic) $>$ Densidade (DS) > Porosidade Total (PT), evidenciada pelos valores pela proximidade entre as suas medianas e médias. Observou-se que os resultados, mostraram bem acima da média da distribuição normal, como segue: (Mi-Raiz >> Mi-Solo > Rr-Raiz >Solo). A mediana aponta predominância de ausência positiva na produção do algodoeiro (Tabela 1).

A estatística descritiva de nutrientes foliares do algodoeiro, da produção e da qualidade da fibra de algodão, apresentada na Tabela 2, aponta que houve menores dispersões dos dados de unf e mat, em relação às médias, enquanto que Mo e Mn se destacaram pelos maiores coeficientes de variação. Os dados das variáveis N, K, ELG, SCI, SFI, UNF, MAT, STR e UHM quase se ajustaram à normalidade, com base na curtose e assimetria, bem como pela semelhança entre as suas medianas e médias. A análise da curtose permitiu constatar que as médias de algumas variáveis estiveram acima das médias da distribuição normal, como segue: $\mathrm{RD}>\mathrm{Fe}>\mathrm{Ca}>\mathrm{B}>\mathrm{Cu}>\mathrm{Mn}$. Os nutrientes N, P, K, Mg, $\mathrm{S}, \mathrm{B}, \mathrm{Zn}, \mathrm{Cu}$ e $\mathrm{Mn}$ apresentaram larga amplitude, desde teores abaixo dos níveis considerados baixos até níveis considerados excessivos, mas o Ca e o Fe não atingiram os teores considerados baixos.

Tabela 1. Resumo de correlações entre os atributos físicos do solo.

\begin{tabular}{ccccccc}
\hline $\begin{array}{c}\text { Atributo } \\
\text { físico }\end{array}$ & Mac & PT & DS & Argila & Silte & Areia \\
\hline Mic & $(-) \mathrm{M}$ & $(+) \mathrm{B}$ & $(-) \mathrm{M}$ & $(+) \mathrm{M}$ & $(+) \mathrm{M}$ & $(-) \mathrm{M}$ \\
Mac & & $(-) \mathrm{B}$ & $(-) \mathrm{B}$ & $(-) \mathrm{B}$ & $(-) \mathrm{A}$ & $(+) \mathrm{M}$ \\
PT & & & $(-) \mathrm{M}$ & $(+) \mathrm{M}$ & $(+) \mathrm{B}$ & $(-) \mathrm{M}$ \\
DS & & & $(-) \mathrm{M}$ & ns & $(+) \mathrm{B}$ \\
Argila & & & & $(+) \mathrm{M}$ & $(-) \mathrm{A}$ \\
Silte & & & & & & $(-) \mathrm{M}$ \\
\hline
\end{tabular}

+: correlação positiva; -: correlação negativa; ns: correlação não significativa; A: correlação relativamente alta; M: correlação relativamente média; B: correlação relativamente baixa; Mic: microporosidade; Mac: macroporosidade; PT: porosidade total; DS: densidade do solo.

Fonte: Autores (2021). 
Tabela 2. Estatística descritiva dos dados dos nutrientes foliares do algodoeiro, da produção e dos atributos de qualidade da fibra de algodão $(n=171)$.

\begin{tabular}{|c|c|c|c|c|c|c|c|c|}
\hline Variável & Mín & Máx & Média & DP* & Mediana & Assimetria & Curtose & $\mathbf{C V}$ \\
\hline $\mathrm{N}$ & 21,84 & 53,14 & 39,08 & 5,57 & 39,31 & $-0,33$ & 0,01 & 14,25 \\
\hline $\mathrm{P}$ & 1,49 & 9,09 & 3,11 & 0,99 & 2,89 & 2,11 & 8,28 & 31,93 \\
\hline $\mathrm{K}$ & 4,26 & 31,51 & 15,56 & 4,95 & 14,99 & 0,37 & $-0,01$ & 31,78 \\
\hline $\mathrm{Ca}$ & 18,66 & 79,50 & 31,13 & 6,15 & 31,40 & 2,83 & 21,45 & 19,74 \\
\hline $\mathrm{Mg}$ & 0,96 & 11,76 & 4,42 & 1,73 & 4,11 & 1,11 & 2,37 & 39,07 \\
\hline $\mathrm{S}$ & 1,66 & 20,33 & 6,95 & 3,29 & 6,41 & 1,45 & 2,76 & 47,27 \\
\hline B & 23,60 & 271,99 & 66,80 & 29,52 & 60,87 & 3,12 & 16,00 & 44,20 \\
\hline $\mathrm{Zn}$ & 8,51 & 82,51 & 25,64 & 10,10 & 23,11 & 1,86 & 5,92 & 39,41 \\
\hline $\mathrm{Cu}$ & 3,04 & 19,21 & 5,96 & 1,85 & 5,64 & 2,83 & 15,86 & 31,13 \\
\hline Mn & 5,86 & 584,63 & 81,16 & 88,66 & 54,61 & 3,50 & 12,00 & 109,24 \\
\hline $\mathrm{Fe}$ & 57,11 & 478,00 & 101,94 & 49,26 & 90,45 & 5,47 & 38,01 & 48,33 \\
\hline Mo & 0,04 & 5,44 & 0,98 & 1,06 & 0,61 & 2,00 & 4,16 & 108,03 \\
\hline $\mathrm{Ni}$ & 0,03 & 1,56 & 0,45 & 0,25 & 0,40 & 1,39 & 3,09 & 55,30 \\
\hline $\mathrm{PF}+\mathrm{C}$ & 2,50 & 164,50 & 47,60 & 27,32 & 45,00 & 0,87 & 1,45 & 57,40 \\
\hline $\mathrm{RF}$ & 33,85 & 49,15 & 42,11 & 1,90 & 42,05 & $-0,34$ & 2,13 & 4,50 \\
\hline PF & 1,06 & 69,94 & 19,23 & 11,11 & 18,42 & 1,00 & 2,22 & 57,80 \\
\hline SCI & 103,00 & 167,00 & 139,38 & 12,84 & 139 & $-0,25$ & $-0,26$ & 9,21 \\
\hline UHM & 1,03 & 1,28 & 1,15 & 0,05 & 1,15 & 0,11 & $-0,75$ & 4,65 \\
\hline UNF & 78,70 & 85,20 & 82,43 & 1,28 & 82,50 & $-0,24$ & $-0,31$ & 1,55 \\
\hline SFI & 5,90 & 12,40 & 8,62 & 1,27 & 8,40 & 0,60 & 0,26 & 14,72 \\
\hline STR & 26,20 & 35,30 & 30,80 & 1,95 & 30,80 & 0,05 & $-0,57$ & 6,35 \\
\hline MAT & 83,00 & 88,00 & 85,72 & 1,20 & 86,00 & $-0,06$ & $-0,37$ & 1,40 \\
\hline ELG & 5,50 & 7,20 & 6,19 & 0,34 & 6,20 & 0,32 & 0,07 & 5,52 \\
\hline RD & 37,60 & 84,70 & 80,31 & 4,58 & 81,30 & $-5,82$ & 47,00 & 5,70 \\
\hline$+b$ & 6,30 & 12,00 & 8,20 & 0,78 & 8,20 & 0,94 & 3,81 & 9,47 \\
\hline Cor & 111,00 & 812,00 & 212,39 & 112,82 & 211,00 & 1,77 & 5,22 & 53,12 \\
\hline MIC & 3,04 & 5,05 & 5,05 & 0,44 & 4,04 & $-0,08$ & $-0,30$ & 10,91 \\
\hline
\end{tabular}

*DP: desvio padrão (\%); N: nitrogênio $\left(\mathrm{g} \mathrm{kg}^{-1}\right)$; P: fósforo $\left(\mathrm{g} \mathrm{kg}^{-1}\right)$; K: potássio $\left(\mathrm{g} \mathrm{kg}^{-1}\right)$; Ca: cálcio $\left(\mathrm{g} \mathrm{kg}^{-1}\right) ; \mathrm{Mg}$ : magnésio $\left(\mathrm{g} \mathrm{kg}^{-1}\right)$; $\mathrm{S}$ : enxofre $\left(\mathrm{g} \mathrm{kg}^{-1}\right)$; B: boro $\left(\mathrm{mg} \mathrm{kg}^{-1}\right)$; $\mathrm{Zn}$ : zinco $\left(\mathrm{mg} \mathrm{kg}^{-1}\right)$; Cu: cobre $\left(\mathrm{mg} \mathrm{kg}^{-1}\right)$; Mn: manganês $\left(\mathrm{mg} \mathrm{kg}^{-1}\right)$; Fe: ferro (mg kg$\left.{ }^{-1}\right)$; Mo: molibdênio ( $\left.\mathrm{mg} \mathrm{kg}^{-1}\right)$; Ni: níquel ( $\left.\mathrm{mg} \mathrm{kg}^{-1}\right)$; $\mathrm{PF}+\mathrm{C}$ : produção de algodão em caroço $\left(\mathrm{g}\right.$ planta $\left.^{-1}\right)$; RF: rendimento da fibra de algodão (\%); PF: produção da fibra de algodão $\left(\mathrm{g} \mathrm{planta}^{-1}\right)$; SCI: índice de fiabilidade (\%);

Fonte: Autores (2021).

\subsection{Nutrientes foliares do algodoeiro e produção de algodão em caroço, produção de fibra e rendimento de fibra}

A correlação canônica demonstrou que os nutrientes foliares explicaram, através dos seus dois primeiros eixos, respectivamente, 99,83 e 0,16\% das variações na produção de algodão em caroço, bem como na produção e no rendimento da fibra de algodão. Em relação os nutrientes foliares os mesmos afetaram os aspectos produtivos do algodoeiro, exceto Ca e N, que não influenciaram a produção de algodão em caroço e a produção de fibra, respectivamente (Figura 1). 
Figura 1. Análise de correlação canônica entre os nutrientes foliares do algodoeiro e a produção de algodão em caroço, a produção e o rendimento de fibra. Os nutrientes foliares do algodoeiro estão representados pelos vetores de nitrogênio (N), fósforo $(\mathrm{P})$, potássio $(\mathrm{K})$, cálcio $(\mathrm{Ca})$, magnésio $(\mathrm{Mg})$, enxofre $(\mathrm{S})$, ferro $(\mathrm{Fe})$, cobre $(\mathrm{Cu})$, zinco $(\mathrm{Zn})$, manganês $(\mathrm{Mn})$, boro (B), molibdênio (Mo) e níquel (Ni). Os pontos representam a produção de algodão em caroço (PF+C), o rendimento de fibra de algodão (RF) e a produção de fibra de algodão (PF).

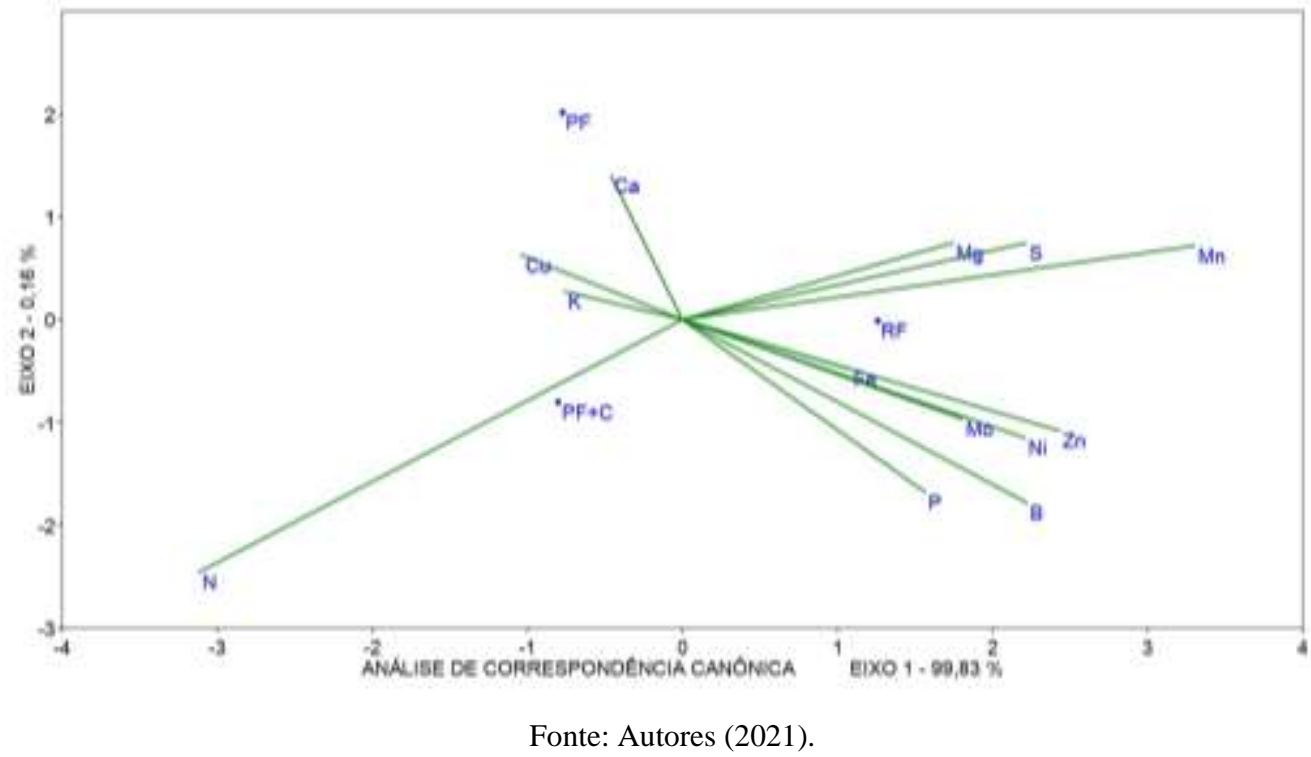

De acordo com os dados apresentados em resumo de correlações entre os nutrientes foliares do algodoeiro e a produção de algodão, entretanto, em todos os casos encontramos a mesma correlação de cada nutriente, positiva ou negativa, com a produção de algodão em caroço e a produção de fibra, mas correlação contrária com o rendimento de fibra, exceto de $\mathrm{N}$ e P (Tabela 3).

Tabela 3. Resumo de correlações entre os nutrientes foliares do algodoeiro e a produção de algodão.

\begin{tabular}{cccc}
\hline Nutriente foliar & PF+C & RF & PF \\
\hline N & $(+) \mathrm{A}$ & $(-) \mathrm{M}$ & $\mathrm{ns}$ \\
$\mathrm{P}$ & $(-) \mathrm{B}$ & $(+) \mathrm{B}$ & $(-) \mathrm{M}$ \\
$\mathrm{K}$ & $(+) \mathrm{B}$ & $(-) \mathrm{B}$ & $(+) \mathrm{B}$ \\
$\mathrm{Ca}$ & $\mathrm{ns}$ & $(-) \mathrm{M}$ & $(-) \mathrm{B}$ \\
$\mathrm{Mg}$ & $(-) \mathrm{M}$ & $(+) \mathrm{M}$ & $(-) \mathrm{B}$ \\
$\mathrm{S}$ & $(-) \mathrm{M}$ & $(+) \mathrm{M}$ & $(-) \mathrm{M}$ \\
$\mathrm{B}$ & $(-) \mathrm{M}$ & $(+) \mathrm{M}$ & $(-) \mathrm{M}$ \\
$\mathrm{Zn}$ & $(-) \mathrm{M}$ & $(+) \mathrm{M}$ & $(+) \mathrm{B}$ \\
$\mathrm{Cu}$ & $(+) \mathrm{B}$ & $(-) \mathrm{B}$ & $(-) \mathrm{B}$ \\
$\mathrm{Mn}$ & $(-) \mathrm{M}$ & $(+) \mathrm{A}$ & $(-) \mathrm{B}$ \\
$\mathrm{Fe}$ & $(-) \mathrm{B}$ & $(+) \mathrm{B}$ & $(-) \mathrm{M}$ \\
$\mathrm{Mo}$ & $(-) \mathrm{M}$ & $(+) \mathrm{M}$ & $(-) \mathrm{M}$ \\
$\mathrm{Ni}$ & $(-) \mathrm{M}$ & $(+) \mathrm{M}$ & \\
\hline
\end{tabular}

+: correlação positiva; -: correlação negativa; ns: correlação não significativa; A: correlação relativamente alta; M: correlação relativamente média; B: correlação relativamente baixa; PF+C: produção de algodão em caroço; RF: rendimento de fibra de algodão; PF: produção de fibra de algodão.

Fonte: Autores (2021)

\subsection{Nutrientes foliares do algodoeiro e qualidade da fibra de algodão}

A correlação canônica mostrou que os nutrientes foliares explicaram, através dos seus dois primeiros eixos, respectivamente 98,25 e 1,30\% das variações, na qualidade da fibra de algodão. Houve influência notória dos nutrientes 
foliares nos mais diferentes atributos de qualidade da fibra de algodão, positiva com intensidade variada. No entanto, alguns nutrientes foliares não afetaram determinados atributos de qualidade da fibra de algodão (Figura 02; Tabela 4,Tabela 5 e Tabela 6).

Tabela 4. Demonstrativo da influência dos nutrientes foliares do algodoeiro, na produção de algodão.

\begin{tabular}{cccc}
$\begin{array}{c}\text { Variável de } \\
\text { produção }\end{array}$ & Influência positiva & Influência negativa & Sem influência \\
\hline $\mathrm{PF}+\mathrm{C}$ & $\mathrm{N}>>(\mathrm{K}=\mathrm{Cu})>\mathrm{Ca}$ & $\mathrm{Mn}>(\mathrm{S}=\mathrm{Mg})>\mathrm{Zn}>\mathrm{Ni}>$ & $\mathrm{Ca}$ \\
$\mathrm{RF}$ & $\mathrm{Mn}>>\mathrm{Zn}>\mathrm{S}>\mathrm{Ni}>\mathrm{Mg}>\mathrm{B})>\mathrm{P}>\mathrm{Fe}$ \\
$\mathrm{Mo}>\mathrm{B}>\mathrm{P}>\mathrm{Fe}$ & $\mathrm{N}>(\mathrm{K}=\mathrm{Cu})>\mathrm{Ca}$ & - \\
$\mathrm{PF}$ & $\mathrm{Ca}>\mathrm{Cu}>\mathrm{K}$ & $(\mathrm{P}=\mathrm{B})>(\mathrm{Ni}=\mathrm{Zn})>\mathrm{Mo}>$ & $\mathrm{N}$ \\
\hline
\end{tabular}

PF+C: produção de algodão em caroço; RF: rendimento de fibra de algodão; PF: produção de fibra de algodão.

Fonte: Autores (2021).

Figura 2. Análise de correlação canônica entre os nutrientes foliares do algodoeiro e a qualidade de fibra. Os nutrientes foliares do algodoeiro estão representados pelos vetores. Os pontos representam maturidade da fibra (MAT), finura da fibra (MIC), índice de fiabilidade (SCI), índice de fibras curtas (SFI), e alongamento da fibra até à sua ruptura (ELG), reflectância da fibra $(\mathrm{Rd})$, uniformidade do comprimento da fibra (UNF), grau de amarelecimento da fibra (+b), resistência da fibra à ruptura (STR), cor da fibra (Cor) e comprimento médio da fibra (UHM).

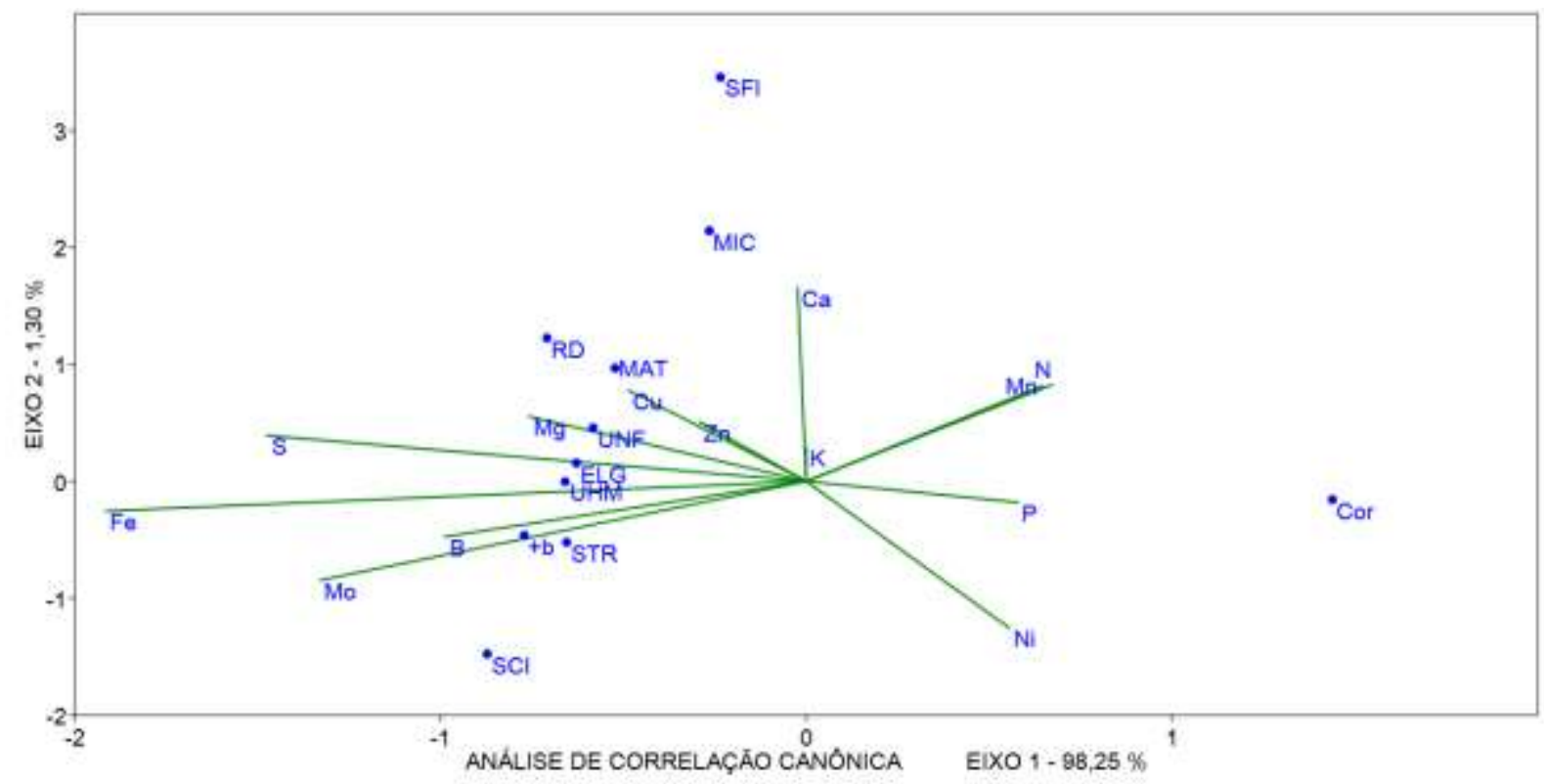

Fonte: Autores (2021). 
Tabela 5. Resumo de correlações entre os nutrientes foliares do algodoeiro e os atributos de qualidade da fibra de algodão.

\begin{tabular}{cccccccccccc}
\hline $\begin{array}{c}\text { Nutriente } \\
\text { foliar }\end{array}$ & SCI & UHM & UNF & SFI & STR & MAT & ELG & RD & +b & Cor & MIC \\
\hline $\mathrm{N}$ & $(-) \mathrm{M}$ & $(-) \mathrm{M}$ & $(-) \mathrm{M}$ & $\mathrm{ns}$ & $(-) \mathrm{A}$ & $(-) \mathrm{B}$ & $(-) \mathrm{M}$ & $(-) \mathrm{B}$ & $(-) \mathrm{A}$ & $(+) \mathrm{B}$ & $\mathrm{ns}$ \\
$\mathrm{P}$ & $(-) \mathrm{B}$ & $(-) \mathrm{A}$ & $(-) \mathrm{M}$ & $(-) \mathrm{B}$ & $(-) \mathrm{M}$ & $(-) \mathrm{M}$ & $(-) \mathrm{A}$ & $(-) \mathrm{M}$ & $(-) \mathrm{M}$ & $(+) \mathrm{M}$ & $(-) \mathrm{B}$ \\
$\mathrm{K}$ & $\mathrm{ns}$ & $\mathrm{ns}$ & $\mathrm{ns}$ & $\mathrm{ns}$ & $\mathrm{ns}$ & $\mathrm{ns}$ & $\mathrm{ns}$ & $\mathrm{ns}$ & $\mathrm{ns}$ & $\mathrm{ns}$ & $\mathrm{ns}$ \\
$\mathrm{Ca}$ & $(-) \mathrm{M}$ & $\mathrm{ns}$ & $(+) \mathrm{B}$ & $(+) \mathrm{B}$ & $(-) \mathrm{B}$ & $(+) \mathrm{B}$ & $\mathrm{ns}$ & $(+) \mathrm{B}$ & $(-) \mathrm{B}$ & $\mathrm{ns}$ & $(+) \mathrm{M}$ \\
$\mathrm{Mg}$ & $(+) \mathrm{B}$ & $(+) \mathrm{M}$ & $(+) \mathrm{M}$ & $(+) \mathrm{B}$ & $(+) \mathrm{B}$ & $(+) \mathrm{M}$ & $(+) \mathrm{M}$ & $(+) \mathrm{M}$ & $(+) \mathrm{B}$ & $(-) \mathrm{M}$ & $(+) \mathrm{B}$ \\
$\mathrm{S}$ & $(+) \mathrm{B}$ & $(+) \mathrm{A}$ & $(+) \mathrm{M}$ & $\mathrm{ns}$ & $(+) \mathrm{M}$ & $(+) \mathrm{M}$ & $(+) \mathrm{A}$ & $(+) \mathrm{M}$ & $(+) \mathrm{M}$ & $(-) \mathrm{B}$ & $(+) \mathrm{B}$ \\
$\mathrm{B}$ & $(+) \mathrm{M}$ & $(+) \mathrm{M}$ & $(+) \mathrm{M}$ & $\mathrm{ns}$ & $(+) \mathrm{A}$ & $(+) \mathrm{B}$ & $(+) \mathrm{M}$ & $(+) \mathrm{B}$ & $(+) \mathrm{A}$ & $(-) \mathrm{M}$ & $\mathrm{ns}$ \\
$\mathrm{Zn}$ & $(+) \mathrm{B}$ & $(+) \mathrm{B}$ & $(+) \mathrm{M}$ & $(+) \mathrm{B}$ & $(+) \mathrm{B}$ & $(+) \mathrm{M}$ & $(+) \mathrm{B}$ & $(+) \mathrm{M}$ & $(+) \mathrm{B}$ & $(-) \mathrm{B}$ & $(+) \mathrm{M}$ \\
$\mathrm{Cu}$ & $(+) \mathrm{B}$ & $(+) \mathrm{M}$ & $(+) \mathrm{M}$ & $(+) \mathrm{B}$ & $(+) \mathrm{B}$ & $(+) \mathrm{A}$ & $(+) \mathrm{M}$ & $(+) \mathrm{A}$ & $(+) \mathrm{B}$ & $(-) \mathrm{B}$ & $(+) \mathrm{B}$ \\
$\mathrm{Mn}$ & $(-) \mathrm{M}$ & $(-) \mathrm{M}$ & $(-) \mathrm{M}$ & $\mathrm{ns}$ & $(-) \mathrm{A}$ & $(-) \mathrm{B}$ & $(-) \mathrm{M}$ & $(-) \mathrm{B}$ & $(-) \mathrm{A}$ & $(+) \mathrm{B}$ & $\mathrm{ns}$ \\
$\mathrm{Fe}$ & $(+) \mathrm{M}$ & $(+) \mathrm{A}$ & $(+) \mathrm{M}$ & $\mathrm{ns}$ & $(+) \mathrm{M}$ & $(+) \mathrm{M}$ & $(+) \mathrm{M}$ & $(+) \mathrm{M}$ & $(+) \mathrm{M}$ & $(-) \mathrm{M}$ & $(+) \mathrm{B}$ \\
$\mathrm{Mo}$ & $(+) \mathrm{M}$ & $(+) \mathrm{M}$ & $(+) \mathrm{B}$ & $\mathrm{ns}$ & $(+) \mathrm{A}$ & $(+) \mathrm{B}$ & $(+) \mathrm{M}$ & $(+) \mathrm{B}$ & $(+) \mathrm{A}$ & $(-) \mathrm{M}$ & $\mathrm{ns}$ \\
$\mathrm{Ni}$ & $(-) \mathrm{B}$ & $(-) \mathrm{M}$ & $(-) \mathrm{M}$ & $(-) \mathrm{M}$ & $(-) \mathrm{B}$ & $(-) \mathrm{A}$ & $(-) \mathrm{M}$ & $(-) \mathrm{A}$ & $(-) \mathrm{B}$ & $(+) \mathrm{B}$ & $(-) \mathrm{M}$ \\
\hline
\end{tabular}

+: correlação positiva; -: correlação negativa; ns: correlação não significativa; A: correlação relativamente alta; M: correlação relativamente média; B: correlação relativamente baixa; MAT: maturidade da fibra; MIC: finura da fibra; SCI: índice de fiabilidade; SFI: índice de fibras curtas; ELG: alongamento da fibra até à sua ruptura; RD: reflectância da fibra; UNF: uniformidade do comprimento da fibra; +b: grau de amarelecimento da fibra; STR: resistência da fibra à ruptura; Cor: cor da fibra; UHM: comprimento médio da fibra.

Fonte: Autores (2021).

Tabela 6. Demonstrativo da influência dos nutrientes foliares do algodoeiro, nos atributos de qualidade da fibra de algodão.

\begin{tabular}{|c|c|c|c|}
\hline Atributo & Influência positiva & Influência negativa & Influência desprezível \\
\hline SCI & $\begin{array}{c}\mathrm{Mo}>\mathrm{Fe}>\mathrm{B}>\mathrm{S}> \\
\mathrm{Mg}>\mathrm{Cu}>\mathrm{Zn}\end{array}$ & $(\mathrm{Mn}=\mathrm{N})>\mathrm{P}>\mathrm{Ca}>\mathrm{Ni}$ & $\mathrm{K}$ \\
\hline UHM & $\begin{array}{c}\mathrm{Fe}>\mathrm{S}>\mathrm{Mo}>\mathrm{B}> \\
\mathrm{Mg}>\mathrm{Cu}>\mathrm{Zn}\end{array}$ & $\mathrm{P}>(\mathrm{Mn}=\mathrm{N})>\mathrm{Ni}$ & $\mathrm{K}, \mathrm{Ca}$ \\
\hline UNF & $\begin{array}{c}\mathrm{Mg}>\mathrm{S}>\mathrm{Fe}>\mathrm{Cu}> \\
\mathrm{Mo}>\mathrm{B}>\mathrm{Zn}>\mathrm{Ca}\end{array}$ & $\mathrm{P}>\mathrm{Ni}>(\mathrm{Mn}=\mathrm{N})$ & $\mathrm{K}$ \\
\hline SFI & $\mathrm{Ca}>(\mathrm{N}=\mathrm{Mn})>\mathrm{Cu}>\mathrm{Zn}$ & $\mathrm{Ni}>\mathrm{Mo}>\mathrm{P}$ & $\begin{array}{l}\mathrm{K}, \mathrm{N}, \mathrm{S}, \mathrm{B}, \\
\mathrm{Mn}, \mathrm{Fe}, \mathrm{Mo}\end{array}$ \\
\hline STR & $\begin{array}{c}\mathrm{Mo}>\mathrm{Fe}>\mathrm{B}>\mathrm{S}> \\
\mathrm{Mg}>\mathrm{Cu}>\mathrm{Zn}\end{array}$ & $(\mathrm{Mn}=\mathrm{N})>\mathrm{P}>\mathrm{Ni}>\mathrm{Ca}$ & $\mathrm{K}$ \\
\hline MAT & $\begin{array}{c}\mathrm{Cu}>(\mathrm{S}=\mathrm{Fe})>\mathrm{Mg}> \\
\mathrm{Zn}>\mathrm{Mo}>\mathrm{Ca}>\mathrm{B}\end{array}$ & $\mathrm{Ni}>(\mathrm{P}=\mathrm{Mn}=\mathrm{N})$ & $\mathrm{K}$ \\
\hline ELG & $\begin{array}{c}\mathrm{S}>\mathrm{Fe}>\mathrm{Mg}>\mathrm{Mo}> \\
\quad \mathrm{B}>\mathrm{Cu}>\mathrm{Zn}\end{array}$ & $\mathrm{P}>(\mathrm{Mn}=\mathrm{N})>\mathrm{Ni}$ & $\mathrm{K}, \mathrm{Ca}$ \\
\hline $\mathrm{RD}$ & $\begin{array}{c}(\mathrm{Cu}=\mathrm{S}=\mathrm{Fe})>\mathrm{Mg}> \\
\mathrm{Mo}>\mathrm{Ca}>\mathrm{Zn}>\mathrm{B}\end{array}$ & $\mathrm{Ni}>(\mathrm{P}=\mathrm{Mn}=\mathrm{N})$ & $\mathrm{K}$ \\
\hline$+\mathrm{b}$ & $\begin{array}{c}\mathrm{Mo}>\mathrm{Fe}>\mathrm{B}>\mathrm{S}> \\
\mathrm{Mg}>\mathrm{Cu}>\mathrm{Zn}\end{array}$ & $(\mathrm{Mn}=\mathrm{N})>\mathrm{P}>\mathrm{Ni}>\mathrm{Ca}$ & $\mathrm{K}$ \\
\hline Cor & $\mathrm{Ni}>(\mathrm{Mn}=\mathrm{N})>\mathrm{Ni}$ & $\begin{array}{c}(\mathrm{S}=\mathrm{Fe})>\mathrm{Mo}>\mathrm{B}> \\
\mathrm{Mg}>\mathrm{Cu}>\mathrm{Zn}\end{array}$ & $\mathrm{K}, \mathrm{Ca}$ \\
\hline MIC & $\begin{array}{c}\mathrm{Ca}>(\mathrm{N}=\mathrm{Mn})> \\
\mathrm{Cu}>\mathrm{Zn}\end{array}$ & $\mathrm{Ni}>\mathrm{Mo}>\mathrm{P}$ & $\mathrm{K}, \mathrm{N}, \mathrm{B}, \mathrm{Mn}, \mathrm{Mo}$ \\
\hline
\end{tabular}

SCI: índice de fiabilidade; UHM: comprimento médio da fibra; UNF: uniformidade do comprimento da fibra; SFI: índice de fibras curtas; STR: resistência da fibra à ruptura; MAT: maturidade da fibra; ELG: alongamento da fibra até à sua ruptura; RD: reflectância da fibra; +b: grau de amarelamento da fibra; Cor: cor da fibra; MIC: finura da fibra. *Efeitos não expressivo. Fonte: Autores (2021).

No que diz respeito à concentração de nutrientes foliares, praticamente não houve diferença entre as classes Severa e Moderada, porém apresentou menor diferença entre as classes Ausente e Muito Severa. Todas as classes estão próximas entre si e se identificam com Mg. As classes Ausente e Muito Severa se caracterizaram por P, enquanto que as classes Severa e Moderada não diferiram entre si pelo teor de S. Os atributos MS-Raiz e Mn não tiveram correlação com as classes de danos ao 
algodoeiro. É provável que MS-Raiz não tenha se correlacionado com as classes de danos ao algodoeiro, porque as raízes coletadas não foram retiradas de volume padronizado de solo (Figura 3).

Figura 3. Análise de correspondência entre os nutrientes foliares e as classes de danos ao algodoeiro. Níveis de classes: Ausente, Moderada, Severa e Muito Severa. MS-Raiz: matéria seca de raiz.

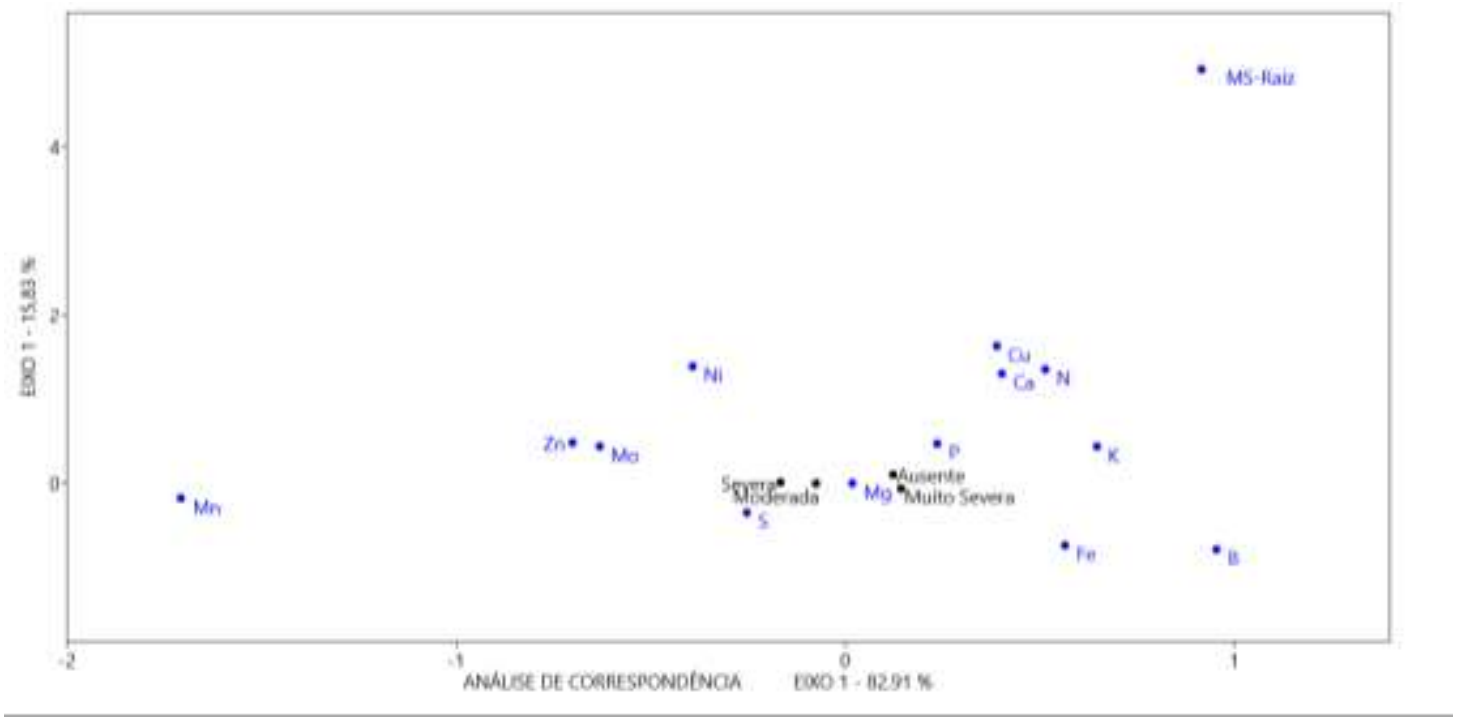

Fonte: Autores (2021).

A influência da alteração dos teores de nutrientes foliares, no incremento de produção da fibra de algodão, varia em função da produção de algodão em caroço, do rendimento de fibra e da produção de fibra (Tabela 7).

Tabela 7. Demonstrativo de influência da alteração de teores dos nutrientes foliares, no incremento da produção de fibra de algodão.

\begin{tabular}{|c|c|c|c|c|c|c|}
\hline \multirow{3}{*}{$\begin{array}{l}\text { Variável de } \\
\text { produção }\end{array}$} & \multicolumn{3}{|c|}{ Redução de nutriente foliar } & \multicolumn{3}{|c|}{ Elevação de nutriente foliar } \\
\hline & \multicolumn{3}{|c|}{ Nível de influência } & \multicolumn{3}{|c|}{ Nível de influência } \\
\hline & Alto & Médio & Baixo & Alto & Médio & Baixo \\
\hline $\mathrm{PF}+\mathrm{C}$ & - & $\begin{array}{c}\mathrm{Mg}, \mathrm{S}, \mathrm{B}, \\
\mathrm{Zn}, \mathrm{Mn}, \mathrm{Mo}, \\
\mathrm{Ni}\end{array}$ & $\mathrm{P}, \mathrm{Fe}$ & $\mathrm{N}$ & - & $\mathrm{K}, \mathrm{Cu}$ \\
\hline $\mathrm{RF}$ & - & $\mathrm{N}, \mathrm{Ca}$ & K & Mn & $\begin{array}{l}\mathrm{Mg}, \mathrm{S}, \mathrm{B}, \\
\mathrm{Zn}, \mathrm{Mo}, \mathrm{Ni}\end{array}$ & $\mathrm{P}, \mathrm{Fe}$ \\
\hline $\mathrm{PF}$ & - & $\begin{array}{l}\mathrm{P}, \mathrm{B}, \mathrm{Zn}, \\
\mathrm{Mo}, \mathrm{Ni}\end{array}$ & $\begin{array}{l}\mathrm{Mg}, \mathrm{S} \\
\mathrm{Mn}, \mathrm{Fe}\end{array}$ & - & $\mathrm{Ca}$ & $\mathrm{K}, \mathrm{Cu}$ \\
\hline
\end{tabular}

PF+C: produção de algodão em caroço; RF: rendimento de fibra de algodão; PF: produção de fibra de algodão. Fonte: Autores (2021).

A influência da alteração dos teores de nutrientes foliares, na melhoria da qualidade da fibra de algodão, varia em função do atributo da qualidade da fibra (Tabela 8). 
Tabela 8. Demonstrativo de influência da alteração de teores dos nutrientes foliares, na melhoria de atributos de qualidade da fibra de algodão.

\begin{tabular}{|c|c|c|c|c|c|c|}
\hline \multirow{3}{*}{ Atributo } & \multirow{2}{*}{\multicolumn{3}{|c|}{$\begin{array}{c}\text { Redução de nutriente foliar } \\
\text { Nível de influência }\end{array}$}} & \multirow{2}{*}{\multicolumn{3}{|c|}{$\begin{array}{c}\text { Elevação de nutriente foliar } \\
\text { Nível de influência }\end{array}$}} \\
\hline & & & & & & \\
\hline & Alto & Médio & Baixo & Alto & Médio & Baixo \\
\hline SCI & - & $\mathrm{N}, \mathrm{Ca}, \mathrm{Mn}$ & $\mathrm{Ni}, \mathrm{P}$ & - & $\mathrm{B}, \mathrm{Fe}, \mathrm{Mo}$ & $\begin{array}{l}\mathrm{Mg}, \mathrm{S} \\
\mathrm{Zn}, \mathrm{Cu}\end{array}$ \\
\hline UHM & $\mathrm{P}$ & $\mathrm{N}, \mathrm{Mn}, \mathrm{Ni}$ & - & $\mathrm{S}, \mathrm{Fe}$ & $\begin{array}{l}\mathrm{Mg}, \mathrm{B} \\
\mathrm{Cu}, \mathrm{Mo}\end{array}$ & $\mathrm{Zn}$ \\
\hline UNF & - & $\begin{array}{l}\mathrm{N}, \mathrm{P} \\
\mathrm{Mn}, \mathrm{Ni}\end{array}$ & - & - & $\begin{array}{l}\mathrm{Mg}, \mathrm{S}, \mathrm{B} \\
\mathrm{Zn}, \mathrm{Cu}, \mathrm{Fe}\end{array}$ & $\mathrm{Ca}, \mathrm{Mo}$ \\
\hline SFI & - & - & $\begin{array}{l}\mathrm{Ca}, \mathrm{Mg} \\
\mathrm{Zn}, \mathrm{Cu}\end{array}$ & - & $\mathrm{Ni}$ & $\mathrm{P}$ \\
\hline STR & $\mathrm{N}, \mathrm{Mn}$ & $\mathrm{P}$ & $\mathrm{Ca}, \mathrm{Ni}$ & B, Mo & $\mathrm{S}, \mathrm{Fe}$ & $\begin{array}{l}\mathrm{Mg}, \mathrm{Zn} \\
\mathrm{Cu}\end{array}$ \\
\hline MAT & $\mathrm{Ni}$ & $\mathrm{P}$ & $\mathrm{N}, \mathrm{Mn}$ & $\mathrm{Cu}$ & $\begin{array}{l}\mathrm{Mg}, \mathrm{S} \\
\mathrm{Zn}, \mathrm{Fe}\end{array}$ & $\mathrm{Ca}, \mathrm{B}, \mathrm{Mo}$ \\
\hline ELG & $\mathrm{P}$ & $\mathrm{N}, \mathrm{Mn}, \mathrm{Ni}$ & - & S & $\begin{array}{l}\mathrm{Mg}, \mathrm{B}, \mathrm{Cu}, \\
\mathrm{Fe}, \mathrm{Mo}\end{array}$ & $\mathrm{Zn}$ \\
\hline $\mathrm{RD}$ & $\mathrm{Ni}$ & $\mathrm{P}$ & $\mathrm{N}, \mathrm{Mn}$ & $\mathrm{Cu}$ & $\begin{array}{l}\mathrm{Mg}, \mathrm{S} \\
\mathrm{Zn}, \mathrm{Fe}\end{array}$ & $\mathrm{Ca}, \mathrm{B}, \mathrm{Mo}$ \\
\hline$+b$ & B, Mo & $\mathrm{S}, \mathrm{Fe}$ & $\underset{\mathrm{Cu}}{\mathrm{Mg}, \mathrm{Zn}}$ & $\mathrm{N}, \mathrm{Mn}$ & $\mathrm{P}$ & $\mathrm{Ca}, \mathrm{Ni}$ \\
\hline Cor & - & $\mathrm{P}$ & $\mathrm{N}, \mathrm{Mn}, \mathrm{Ni}$ & - & $\begin{array}{l}\mathrm{Mg}, \mathrm{B} \\
\mathrm{Fe}, \mathrm{Mo}\end{array}$ & $\mathrm{S}, \mathrm{Zn}, \mathrm{Cu}$ \\
\hline MIC & - & $\mathrm{Ni}$ & $\mathrm{P}$ & - & $\mathrm{Ca}, \mathrm{Zn}$ & $\begin{array}{l}\mathrm{Mg}, \mathrm{S} \\
\mathrm{Cu}, \mathrm{Fe}\end{array}$ \\
\hline
\end{tabular}

SCI: índice de fiabilidade; UHM: comprimento médio da fibra; UNF: uniformidade do comprimento da fibra; SFI: índice de fibras curtas; STR: resistência da fibra à ruptura; MAT: maturidade da fibra; ELG: alongamento da fibra até à sua ruptura; RD: reflectância da fibra; +b: grau de amarelamento da fibra; Cor: cor da fibra; MIC: finura da fibra.

Fonte: Autores (2021).

A influência da alteração dos teores de nutrientes foliares, na produção e qualidade da fibra de algodão, varia em função do atributo da qualidade da fibra (Tabela 9).

Tabela 9. Demonstrativo da influência da alteração de teores dos nutrientes foliares, na produção e qualidade da fibra de algodão.

\begin{tabular}{|c|c|c|c|c|}
\hline \multirow{2}{*}{ Alteração } & \multicolumn{2}{|c|}{ Produção de fibra } & \multicolumn{2}{|c|}{ Qualidade da fibra } \\
\hline & Aumenta & Diminui & Melhora & Piora \\
\hline $\begin{array}{l}\text { Redução de nutriente } \\
\text { foliar }\end{array}$ & $\begin{array}{l}\text { P, Ni, Mn, Zn, Fe, } \\
\text { S, Mg, B e Mo }\end{array}$ & - & $\mathrm{P}, \mathrm{Ni}, \mathrm{Mn}, \mathrm{N}$ & $\begin{array}{c}\mathrm{Zn}, \mathrm{Fe}, \mathrm{S} \\
\mathrm{Mg}, \mathrm{B} \text { e } \mathrm{Mo}\end{array}$ \\
\hline $\begin{array}{c}\text { Elevação de nutriente } \\
\text { foliar }\end{array}$ & $\mathrm{Ca}, \mathrm{Cu}, \mathrm{K}$ & $\begin{array}{c}\mathrm{Zn}, \mathrm{Fe}, \mathrm{S}, \\
\mathrm{Mg}, \mathrm{B} \text { e Mo }\end{array}$ & $\begin{array}{c}\mathrm{Ca}, \mathrm{Cu}, \mathrm{Zn}, \mathrm{Fe}, \mathrm{S} \\
\mathrm{Mg}, \mathrm{B} \text { e } \mathrm{Mo}\end{array}$ & - \\
\hline
\end{tabular}

Fonte: Autores (2021).

\section{Discussão}

A pesquisa de campo, foi realizada através de parâmetros quantitativos para se obter resultados qualitativos na produção da fibra de algodão, que são determinados através dos atributos físicos do solo de acordo com Tabela 1 constatamos que: (i) a granulometria de silte é intermediária entre areia e argila, porém mais próxima da argila; (ii) areia está associada à aeração, por participar da formação da macroporosidade; (iii) argila está vinculada à umidade, por formar a microporosidade; (iv) silte também está associado à microporosidade. Maiores níveis de microporosidade são encontrados em solos argilosos (Stolf et al., 2011), possivelmente seja essa a razão pela qual a influência dos resultados esteja associado a solos argilosos, 
como verificaram Starr et al. (1993) e Moore e Lawrence (2013), já que a qualidade da produção de algodão predominaram em solos com mais microporosidade, que pode ser uma explicação para o avanço, em áreas de cultivo de algodão, predominantemente argilosas, que expandiu de 13\% (média calculada para as 6 regiões de Mato Grosso, encontrada por Galbieri e Belot (2016), para 59\% (média calculada apenas para 3 regiões de Mato Grosso, encontrada por no estudo).

O manejo de nutrientes do solo deve obedecer aos teores de nutrientes foliares, que são considerados suficientes para o algodoeiro (Kurihara et al., 2013). Portanto avaliação apropriado de nutrientes do solo, para verificar a qualidade do algodão, depende da análise da sua acumulação nas folhas e da repercussão sobre a produção e a qualidade da fibra de algodão.

Os resultados encontrados neste estudo foram quantificados e comparados com o trabalho de Stolf et al. (2011) demonstraram a elevação da densidade do solo que diminui a porosidade total e a macroporosidade, mas aumenta a microporosidade. Entretanto, encontramos os seguintes contrastes: elevação da densidade do solo reduz a microporosidade, a porosidade total, a microporosidade e a macroporosidade. Verificamos que a compactação do solo reduziu a porosidade total, diminui a macroporosidade e, principalmente, a microporosidade (Tabela 1). Parece razoável que a compactação reduz mais a microporosidade do que a macroporosidade, já que a microporosidade é constituída de minerais de menor granulometria (Tabela 01) e que, por isso, conseguem se juntar mais uns aos outros, em razão da maior superfície de contato entre si.

Através da análise de correlação canônica para avaliar a qualidade do algodão em caroço e fibra, possibilitou explorar adequadamente a realidade dos cultivos de algodão, já que boa parte dos nutrientes foliares apresentou ampla variação de teores, desde abaixo dos níveis baixos até acima dos níveis excessivos (Kurihara et al., 2013), e se tornou mais considerável pelo envolvimento de dados de amostras provenientes das mais variadas condições de solo e de clima, em função de distintas regiões, altitudes e cultivares. Os nutrientes foliares do algodoeiro apresentaram mais de $99 \%$ da variação da produção e da qualidade da fibra de algodão. Os resultados mostraram que um mesmo nutriente pode apresentar efeitos divergentes sobre a produção e a qualidade, da fibra de algodão (Figura 2; Tabelas,2,3, 4 e 5). Constatamos que Ca e Cu elevaram a produção e melhoraram a qualidade, enquanto que $\mathrm{Zn}, \mathrm{Fe}, \mathrm{S}, \mathrm{Mg}, \mathrm{B}$ e Mo reduziram a produção e melhoraram a qualidade, ao passo que $\mathrm{P}$, Ni e Mn reduziram a produção e a qualidade, da fibra de algodão.

No que diz respeito à concentração de nutrientes foliares (Figura 3), praticamente não houve diferença entre as classes Severa e Moderada, mas bem menos diferença entre as classes Ausente e Muito Severa. Todas as classes estão bem próximas entre si e se identificam com Mg. As classes Ausente e Muito Severa se caracterizaram por P, enquanto que as classes Severa e Moderada não diferiram entre si pelo teor de S. A MS-Raiz e Mn não tiveram correlação com as classes de danos ao algodoeiro. É provável que a MS-Raiz não tenha se correlacionado com as classes de danos ao algodoeiro, porque as raízes coletadas não foram retiradas de volume de solo padronizado.

A MS-Raiz não teve correlação com as classes de danos ao algodoeiro, porque não foi colhido todo o sistema radicular das plantas amostradas, muito embora tenha tendido levemente a se associar à classe Severa (Figura 3).

Os nutrientes foliares influenciaram a produção de algodão em caroço, a produção de fibra e o rendimento de fibra (Figura 2; Tabelas 5 e 6), de acordo com várias referências. Os teores de nutrientes foliares são reflexos da fertilidade do solo, que tanto podem aumentar a produção de algodão (Kurt \& Temiz, 2016; Shah et al., 2017; Win et al., 2017; Ali et al., 2018; Ahmad et al., 2019; Ali et al., 2020), não alterar a produção de algodão (Silva et al., 2016; Haliloglu, 2019), ou até reduzir a produção de algodão (Seçer et al., 2016; Ajmal et al., 2018).

Os resultados encontrados nessa pesquisa corroboram com os estudos de Haliloglu (2019) e apontam tendência de que cada nutriente apresenta somente correlação não significativa ou a mesma correlação, seja ela negativa ou positiva, com a produção de algodão em caroço, o rendimento e a produção de fibra (Tabela 5). Entretanto, nesta pesquisa destacou a mesma correlação de cada nutriente, positiva ou negativa, com a produção de algodão em caroço e a produção de fibra, mas correlação contrária com o rendimento de fibra, exceto de $\mathrm{N}$ e P (Figura 1 e 2; Tabela 6). 
Portanto, com a corroboração dos nossos resultados aprestados neste trabalho foi possível demonstrar, que: (i) $\mathrm{K} \mathrm{e} \mathrm{Cu}$ elevam a produção de algodão em caroço; (ii) Ni reduz a produção de algodão em caroço; (iii) Zn e Mn elevam o rendimento de fibra; e (iv) K eleva a produção de fibra.

Em relação aos nutrientes foliares influenciaram na qualidade da fibra de algodão (Figura 2; Tabelas 2 e 3), em consonância com estudo. A fertilidade do solo repercute os teores de nutrientes foliares, que tanto podem melhorar a qualidade da fibra de algodão (Sawan et al., 2008; Chen et al., 2016; Win et al., 2017; Guo et al., 2017; Ahmad et al., 2018; El-Gedwy, 2018; Afzal et al., 2019), como podem não afetar a qualidade da fibra de algodão (Sawan et al.,1993; Dordas, 2009; Temiz et al., 2009; Kurt \& Temiz, 2016; Karademir \& Karademir, 2019), e, ainda, apresentar piora a qualidade da fibra de algodão (Sawan et al., 2008; Seçer et al., 2016; Echer et al., 2020).

Todavia o efeito positivo do K, no comprimento médio da fibra, diferentemente desta pesquisa. Por outro lado, a maioria das citações indicou efeito positivo do $\mathrm{K}$, na resistência da fibra à ruptura e na finura da fibra, em contraste com o nosso estudo e de Temiz et al. (2009), que não encontramos efeito do K sobre a resistência da fibra à ruptura e a finura da fibra. Sendo assim, o manejo adequado do solo deve admitir os níveis economicamente aceitáveis de suas perdas em produção e qualidade de fibra (Mehl et al., 2018),

\section{Conclusão}

A metodologia aplicada neste estudo permitiu registrar a necessidade de mais estudos de efeitos científicos que fortalecerão a composição de atributos físicos do solo na qualidade de produção de algodão.

Fica evidente, no entanto, que a presença dos atores nas pesquisas de elaboração de ferramentas de auxílio aos grandes produtores rurais e condução das metodologias aplicadas influência na decisão final.

Os efeitos da densidade do solo e da porosidade total, na análise de correlação, não foram tão expressivos, enquanto que a microporosidade, macroporosidade, argila, silte e areia revelaram forte influência estatística. Vale destacar que os vetores que representam a densidade do solo, a porosidade total, a microporosidade e a macroporosidade estão fundamentados em resultados de análises repetidas de cada amostra de solo. Portanto ao analisar os resultados evidenciamos que os nutrientes foliares estão diretamente correlacionados com a qualidade e produção do algodão em caroço e da fibra.

A seguinte alteração dos nutrientes foliares pode incrementar a produção e melhorar a qualidade da fibra de algodão, com níveis de influência alto e médio (representados pelas letras $a$ e $m$, sobrescritas):

1) produção de algodão em caroço: elevando $(\mathrm{N})^{\mathrm{a}}$, mas também reduzindo $(\mathrm{Mg}, \mathrm{S}, \mathrm{B}, \mathrm{Zn}, \mathrm{Mn}, \mathrm{Mo}, \mathrm{Ni})^{\mathrm{m}}$;

2) rendimento da fibra de algodão: elevando $(\mathrm{Mn})^{\mathrm{a}} \mathrm{e}(\mathrm{Mg}, \mathrm{S}, \mathrm{B}, \mathrm{Zn}, \mathrm{Mo}, \mathrm{Ni})^{\mathrm{m}}$, bem como reduzindo $(\mathrm{N}, \mathrm{Ca})^{\mathrm{m}}$;

3) produção da fibra de algodão: elevando $(\mathrm{Ca})^{\mathrm{m}}$ e reduzindo $(\mathrm{P}, \mathrm{B}, \mathrm{Zn}, \mathrm{Mo}, \mathrm{Ni})^{\mathrm{m}}$;

4) qualidade da fibra de algodão: elevando ( $\mathrm{Ca}, \mathrm{Cu}, \mathrm{Zn}, \mathrm{Fe}, \mathrm{S}, \mathrm{Mg}, \mathrm{B}$ e $\mathrm{Mo})$ e reduzindo ( $\mathrm{P}, \mathrm{Ni}, \mathrm{Mn}, \mathrm{N})$.

Portanto, o presente estudo apresentou resultados satisfatórios que irão contribuir para pesquisas futuras que abordam as relações dos fatores físicos do solo e a produtividade do algodão em caroço e fibra nas distintas lavouras presentes no país.

\section{Referências}

Afzal, M. N., Tariq, M., Ahmad, M., Mubeen, K., Khan, M. A., Afzal, M. U., \& Ahmad, S. (2019). Dry matter, lint mass and fiber properties of cotton in response to nitrogen application and planting densities. Pakistan journal of agricultural research, 32(2), 229-240.

Ahmad, S., Akhtar, L. H., Iqbal, N., \& Nasim, M. (2009). Short Communication Cotton (Gossypium hirsutum L.) varieties responded differently to foliar applied boron in terms of quality and yield. Soil and Environment, 28, 88-92. 
Ahmad, Z., Anjum, S., Iqbal, M. A. \& Saeed-Ur-Rehman. H. (2018). Foliar applied potassium enhances fibre quality, water relations and yield of cotton. Journal of agricultural research, 56(1), 17-25.

Ahmad, S., Hussain, N., Ahmed, N., \& Zaka, S. M. (2019). Influence of boron nutrition on physiological parameters and productivity of cotton (Gossypium hirsutum L.) crop. Pak j bot, 51(22), 401-408.

Ajmal, F., Akram, M., IqbaL, R. M., Hussain, M., Hassan, M. W., \& Abdullah, M. (2018). Effect of exogenous application of micronutrients on growth and productivity of cotton (Gossypium hirsutum L.) crop. Bioscience journal, 34(2).

Ali, L., Akram, M., Din, M., Rehman, U., Zafar, M., \& Ghafoor A. (2018). Improving the productivity of cotton (Gossypium hirsutum L.) by integrated use of macro and micronutrients under agro-climatic conditions of Bahawalnagar, Pakistan. Int. j. adv. multidiscip. res, 59(11), 37-43.

Barbosa, G. R. M., Asmus, G. L. (2019). Densidade populacional do nematoides-das-lesões-radiculares em cana-de-açúcar em função de cultivares, manejo do palhiço e escarificação do solo. Revista de Agricultura Neotropical, 6(2), 87-94.

Cardoso, M. D. O., Pedrosa, E. M. R., Ferris, H., Rolim, M. M., Vicente, T. D. S., David, M. D. L. (2015). Comparing sugarcane fields and forest fragments: the effect of disturbance on soil physical properties and nematode assemblages. Soil use and management, 31(3), 397-407.

Chen, Y., Li, Y., Hu, D., Zhang, X., Wen, Y., \& Chen, D. (2016). Spatial distribution of potassium uptake across the cotton plant affects fiber length. Field Crops Research, 192, 126-133.

Conab. (2020). Acompanhamento da safra brasileira 2019/2020.

Dordas, C. (2009). Foliar Application of Manganese Increases Seed Yield and Improves Seed Quality of Cotton Grown on Calcareous Soils. Journal of Plant Nutrition, 32(1), 160-176.

Echer, F. R., Cordeiro, C. F. S., \& Torre, E. J. R. (2020). The effects of nitrogen, phosphorus, and potassium levels on the yield and fiber quality of cotton cultivars. Journal of Plant Nutrition, 43(7), 921-932.

Eleyan, S. E. D., Abodahab, A. A., Abdallah, A. M., \& Rabeh, H. A. (2014) Effect of foliar application of manganese and iron on growth characters, yield and fiber properties of some Egyptian cotton cultivars (Gossypium barbadense L ). International Journal of Agriculture and Crop Sciences (IJACS), 7(13), 12831292.

El-gedwy, E. (2018). Response of Some Egyptian Cotton Cultivars to Foliar Spray by Some Microelements. Annals of Agricultural Science, 56(4) 965-974.

Galbieri, R., Fuzatto, M. G., Cia, E., Lüders, R. R., Machado, A. C., \& Boldt, A. F. (2009). Reação de cultivares de algodoeiro a Meloidogyne incognita em condições de campo e casa de vegetação no estado de Mato Grosso. Tropical Plant Pathology, 34(1), 18-23.

Galbieri, R., Belot, J. L. (2016). Nematoides fitoparasitas do algodoeiro nos cerrados brasileiros: Biologia e medidas de controle. Instituto Mato-grossense do Algodão - IMAmt, Cuiabá, Brasil, p. 37-89.

Galbieri, R., Davis, R. F., Scoz, L.B., Belot, J. L., \& Skantar, A. M. (2020). First report of Meloidogyne enterolobii on cotton in Brazil. Plant Disease, 104(8), 2295-2295.

Goulart, A. M. C. (2008). Aspectos gerais sobre nematoides-das-lesões radiculares (gênero Pratylenchus). Boletim de pesquisa e desenvolvimento 219, Embrapa Cerrados, Planaltina, DF, Brasil. 30 p.

Guo, Y., Wang, X., Xu, K., Zhu, Y., \& Li, L. (2003). Effects of Root-knot Nematode on the Absorption Characteristics of Mineral Elements in Ginger [J]. Acta Agriculturae Boreali-occidentalis Sinica.

Haliloglu, H. (2019). The effect of phosphorus and zinc on yield and on some agronomic characteristics of cotton (Gossypium hirsutum L.). Appl Ecol Env Res, 17(2), 2665-2676.

Holguin, C. M., Gerard, P., Mueller, J. D., Khalilian, A., \& Agudelo, P. (2015). Spatial distribution of reniform nematode in cotton as influenced by soil texture and crop rotations. Phytopathology, 105(5), 674-683.

Hotelling, H. (1935). The most predictable criterion. Journal of Educational Psychology, 26, 139-142.

Hotelling, H. (1936). Relations between two sets of variables. Biometrika, 28, 321-377.

Hurchanik, D., Schmitt, D. P., Hue, N. V., \& Sipes, B. S. (2003). Relationship of Meloidogyne konaensis population densities to nutritional status of coffee roots and leaves. Nematropica, 33(1), 55-64.

Jagadeesh, P., Gaur, H. S. (2014). Relationship between population density of root-knot nematode, Meloidogyne graminicola and the growth and nutrient uptake of rice plant. Vegetos, 27(1), 130-138.

Karademir, E., Karademir, C. (2019). Effect of different boron application on cotton yield components and fiber quality properties. Cercetări Agronomice în Moldova, 51(4), 341-352.

Kim, E., Seo, Y., Kim, Y. S., Park, Y., \& Kim, Y. H. (2017). Effects of Soil Textures on Infectivity of Root-Knot Nematodes on Carrot. Plant Pathol. J., 33(1), 66-74.

Kumar, V., Verma, K. K., \& Kumar, A. (2017). Effect of soil organic matter content and soil texture on reproduction of Meloidogyne graminicola on rice. Journal of entomology and zoology studies, 5(5), 1267-1270. 
Kurihara, C. H., Venegas, V. H. A., Neves, J. C. L., Novais, R. F. D., \& Staut, L. A. (2013). Faixas de suficiência para teores foliares de nutrientes em algodão e em soja, definidas em função de índices DRIS. Revista Ceres, 60(3), 412-419.

Kurt, F., \& Gultekintemiz, M. (2016). The response of cotton (Gossypium hirsutum L.) to soil applied elemental sulphur under boron toxicity. Fresenius Environmental Bulletin, 25, 5511-5515.

Malavolta, E., Vitti, G. C., \& Oliveira, S. A. (1997). Avaliação do estado nutricional das plantas: princípios e aplicações. (2a ed.), Potafos.

Ortiz, P. F., Rolim, M. M., Lima, J. L., Pedrosa, E. M., Dantas, M. S., \& Tavares, U.E. (2017). Physical qualities of an Ultisol under sugarcane and Atlantic forest in Brazil. Geoderma regional, 11, 62-70.

Sawan, Z. M., Mahmoud, M. H., \& El-Guibali, A. H. (2008). Influence of potassium fertilization and foliar application of zinc and phosphorus on growth, yield components, yield and fiber properties of Egyptian cotton (Gossypium barbadense L.). Journal of Plant Ecology, 1(4), 259-270.

Seçer, M., Elmaci, Ö. L., \& Ceylan, Ş. (2016). The Effects of Organic Wastes on Soil and Cotton Quality with respect to the Risk of Boron and Heavy Metal Pollution. International Journal of Agronomy, 1-7.

Shah, J. A., Sial, M. A., Abbas, M. (2017). Disparity in growth, yield and fiber quality of cotton genotypes grown under deficient and adequate levels of boron. Pakistan Journal of Agriculture, Agricultural Engineering and Veterinary Sciences, 33(2), 163-176.

Silva, R. A., Santos, J. L., Oliveira, L. S., Soares, M. R. S., \& Santos, S. M. S. (2016). Biostimulants on mineral nutrition and fiber quality of cotton crop. Rev. Brasil. Eng. Agríc. Ambient., 20(12), 1062-1066.

Smith, A. L. (2015). Identification of resistant or tolerant commercial cotton cultivars to the Fusarium wilt root-knot nematode disease complex and the identification of Fusarium oxysporum f. sp. vasinfectum races in Alabama. Auburn: Auburn University. 49p (Thesis of Master of Science).

Statista. (2020). Leading cotton exporting countries in 2019/2020. https:/www.statista.com/statistics/191895/leading-cotton-exporting-countries/ .

Stolf, R., Thurler, Á. D. M., BacchI, O. O. S., \& Reichardt, K. (2011). Method to estimate soil macroporosity and microporosity based on sand content and bulk density. Revista Brasileira de Ciência do Solo, 35(2), 447-459.

Teixeira, P. C., Donagemma, G. K., Fontana, A., \& Teixeira, W. G. (2017). Manual de métodos de análise de solo. (3a ed.), Embrapa.

Temiz, M., Koca, Y. K., Aydin, F., \& karahan, E. (2009). Effect of foliar potassium and micronutrient additions on yield and fiber quality of cotton (Gossypium hirsutum L.). Journal of Food, Agriculture \& Environment, 7(1), 118-122.

Tsialtas, J.T., Shabala, S., Matsi,t. (2016). A prominent role for leaf calcium as a yield and quality determinant in upland cotton (Gossypium hirsutum L.) varieties grown under irrigated mediterranean conditions. J agro crop sci, (3), 161-73.

Verma, K. K., Jain, R. K. (2006). Nutrient Uptake as Affected by Inorganic Fertilizers under Meloidogyne incognita Infested Conditions in Cotton. Indian Journal of Nematology, 36(1) 60-64.

Win, T. Z., Myint, A. K., Ngwe, K., Thein, S. S., \& Khaing, T. T. (2017). Effects of nitrogen and potassium application on plant growth, yield and fiber quality of cotton (Gossypium hirsutum L.). Journal of Agricultural Research, 4(1), 47-55. 